\title{
Detection of Nine M8.0-L0.5 Binaries: The Very Low Mass Binary Population and Its Implications for Brown Dwarf Formation Theories
}

\author{
Laird M. Close, Nick Siegler, \& Melanie Freed \\ Steward Observatory, University of Arizona, 933 North Cherry Avenue, \\ Tucson AZ, USA 85721-0065; lclose@as.arizona.edu
}

\begin{abstract}
Use of the highly sensitive Hōkūpa'a/Gemini curvature wavefront sensor has allowed for the first time direct adaptive optics (AO) guiding on very low mass (VLM) stars with $\mathrm{SpT}=\mathrm{M} 8.0-\mathrm{L} 0.5$. A survey of 39 such objects detected 9 VLM binaries (7 of which were discovered for the first time to be binaries). Most of these systems (55\%) are tight (separation $<5 \mathrm{AU})$ and have similar masses $(\Delta K s<0.8 \mathrm{mag}$; $0.85<q<1.0$ ). However, 2 systems (LHS 2397a, and 2M2331016040618) have large $\Delta K s>2.38$ mag and consist of a VLM star orbited by a much cooler L6.5-L8.5 brown dwarf companion. Based on our initial flux limited ( $K s<12 \mathrm{mag}$ ) survey of 39 M8.0-L0.5 stars (mainly from the sample of Gizis et al. 2000) we find a binary fraction in the range $19 \pm 7 \%$ for M8.0-L0.5 binaries with separations $>2.6 \mathrm{AU}$. This is slightly less than the $32 \pm 9 \%$ measured for more massive (M0-M4) stars over the same separation range (Fischer \& Marcy 1992). It appears M8.0-L0.5 binaries (as well as $\mathrm{L}$ and $\mathrm{T}$ dwarf binaries) have a much smaller semimajor axis distribution peak ( $\sim 4 \mathrm{AU})$ compared to more massive $\mathrm{M}$ and $\mathrm{G}$ stars which have a broad peak at larger $\sim 30 \mathrm{AU}$ separations. We also find no VLM binaries $\left(M_{t o t}<0.18 M_{\odot}\right)$ with separations $>20 \mathrm{AU}$. We find that a velocity "kick" of $\sim 3 \mathrm{~km} / \mathrm{s}$ can reproduce the observed cut-off in the semi-major axis distribution at $\sim 20 \mathrm{AU}$. This kick may have been from the VLM system being ejected from its formation mini-cluster.
\end{abstract}

\section{Introduction}

Since the discovery of Gl 229B by Nakajima et al. (1995) there has been intense interest in the direct detection of brown dwarfs and very low mass (VLM) stars. According to the current models of Burrows et al. (2000) and Chabrier et al. (2000), stars with spectral types of M8.0-L0.5 will be just above the stellar/substellar boundary. However, most fainter companions to such primaries should themselves be substellar. Therefore, a survey of M8.0-L0.5 stars should detect binary systems consisting of VLM primaries with VLM or brown dwarf secondaries.

The binary frequency of M8.0-L0.5 stars is interesting in its own right since little is known about how common M8.0-L0.5 binary systems are. It is not clear currently if the M8.0-L0.5 binary separation distribution is similar to that of 
M0-M4 stars; in fact, there is emerging evidence that very low mass L dwarf binaries tend to have smaller separations but similar binary frequencies as their more massive M and G star counterparts (Martín, Brandner, \& Basri (1999) \& Reid et al. (2001a)).

Despite the strong interest in such very low mass binaries, only 13 such systems have been detected to date. In our paper, Close, Siegler, \& Freed (2002), a detailed history of each these detections is given in the Introduction.

During our adaptive optics survey, we have detected 12 VLM binaries (10 of these are new discoveries) out of 69 VLM stars (30 M6.0-M7.5 \& 39 M8.0-L0.5 stars). Three of these new binary systems (LP415-20, LP475-855, \& 2MASSW J1750129+442404) have M7.0-M7.5 spectral types and are discussed in detail elsewhere (Siegler et al. (2002)) with the other $30 \mathrm{M} 6.0-\mathrm{M} 7.5$ stars. In this paper, we discuss the remaining 9 cooler binaries from the sample of 39 M8.0L0.5 targets observed in our survey (referred herein as M8.0-L0.5 binaries even though they may contain much cooler L1-L7.5 companions; see Table 1 for a complete list of these systems).

Two of these systems (2MASSW J0746425+200032 and 2MASSW J1047127 +402644) were in our sample yet have been imaged previously in the visible by HST and found to be binaries (Reid et al. (2001a); Reid et al. (2002)) however we present the first resolved IR observations of these 2 systems. The seven remaining systems were all discovered to be binaries during this survey. The first 4 systems discovered in our survey (2MASSW J1426316+155701, 2MASSW J2140293+162518, 2MASSW J2206228-204705, and 2MASSW J2331016-040618) have brief descriptions in Close et al. (2002b). However, we have re-analyzed the data from Close et al. (2002b) and include it here for completeness with slightly revised mass estimates. The very interesting M8/L7.5 system LHS 2397a discovered during this survey is discussed in detail elsewhere (Freed, Close \& Siegler (2002)) yet is included here also for completeness. The newly discovered binaries 2MASSW J1127534+741107 and 2MASSW J1311391+803222 are presented here for the first time. See Close, Siegler, \& Freed (2002) for a more complete discussion of these binaries and our observational methods.

These nine M8.0-L0.5 binaries are a significant addition to the other 11 very low mass M8-T6 binaries known to date (Basri \& Martín(1999), Martín, Brandner, \& Basri (1999), Koerner et al. (1999), Martín et al. (2000), Reid et al. (2001a), Potter et al. (2002a), Burgasser et al. (2002)). With relatively short periods our new systems will likely play a significant role in the mass luminosity calibration for VLM stars and brown dwarfs. It is also noteworthy that we can start to characterize this new population of M8.0-L0.5 binaries. We will outline how M8.0-L0.5 binaries are both similar and different from their more massive M \& G counterparts. 


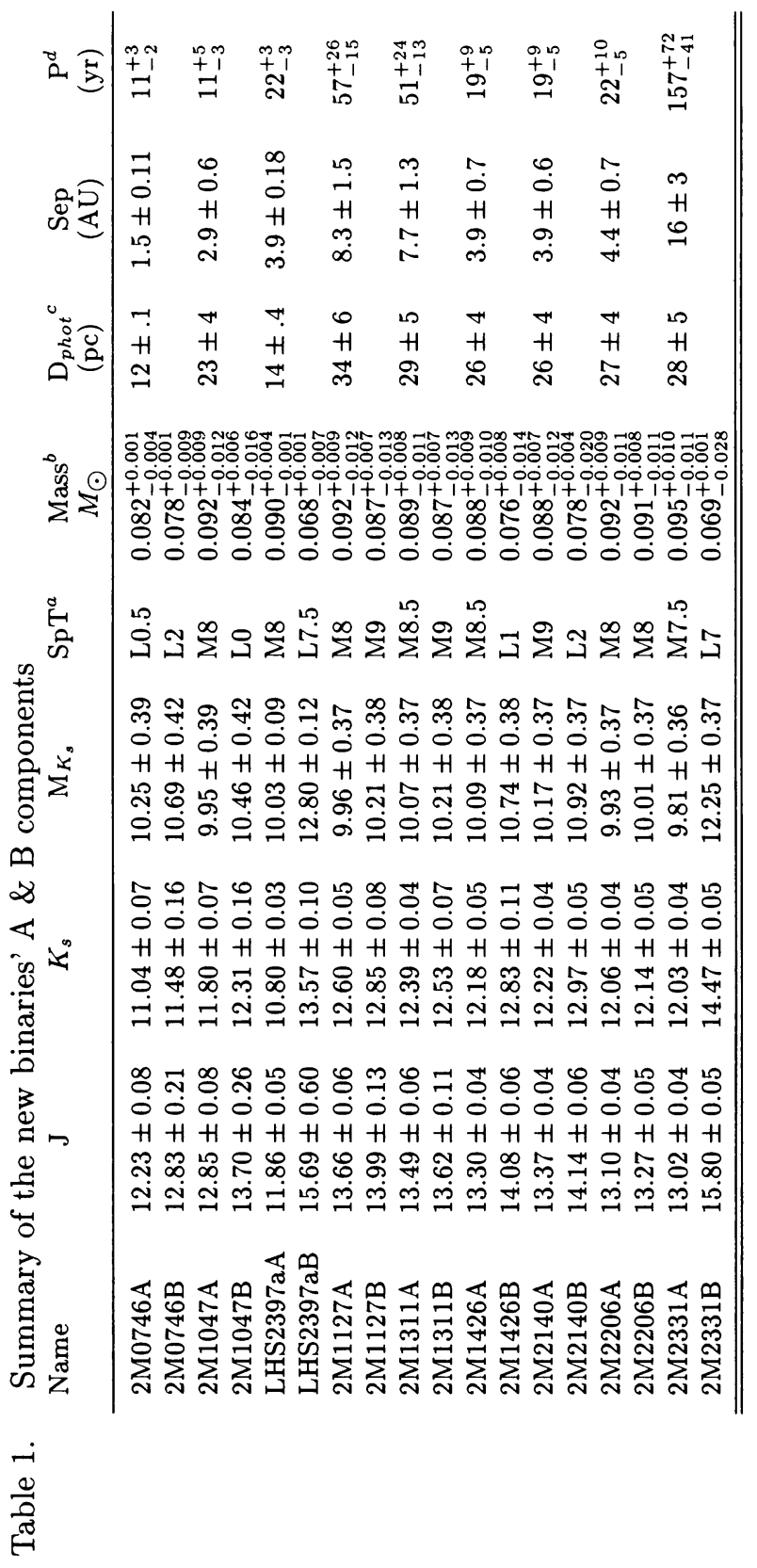

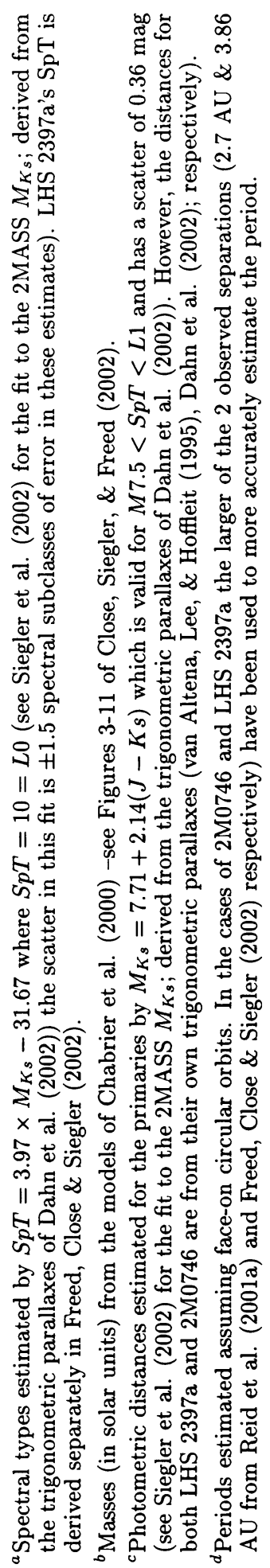




\section{Discussion}

\subsection{The binary frequency of M8.0-L0.5 stars}

We have carried out the largest flux limited $(K s<12)$ high-resolution survey of M8.0-L0.5 primaries. Around these 39 M8.0-L0.5 targets we have detected 9 systems that have companions (see Table 1). Since our survey is flux limited we need to correct for our bias toward detecting equal magnitude binaries that "leak" into our sample from further distances.

More exactly, $88 \%$ of our binary systems are with $29.1 \mathrm{pc}$ and $88 \%$ of our single stars are within $23.4 \mathrm{pc}$. Therefore, we are probing $\sim(29.1 / 23.4)^{3}=1.92$ times more volume with the brighter binaries compared to the single (hence fainter) M8.0-L0.5 stars. Hence, the corrected binary frequency is 9/39/1.92 = $12 \%$.

Of course there are other selection effects due to the instrumental PSF which prevents detection of very faint companions very close to the primaries. We are only sensitive to companions of $\Delta K^{\prime} \lesssim 1 \mathrm{mag}$ at $0.1-0.2^{\prime \prime}$ separations. Much fainter companions $\left(\Delta K^{\prime} \sim 5 \mathrm{mag}\right)$ could be detected at slightly wider $\left(\sim 0.25^{\prime \prime}\right)$ separations, and very low mass companions $(\Delta H \sim 10 \mathrm{mag})$ could be detected at $\sim 1^{\prime \prime}$ separations. Therefore, we are likely not detecting faint $\left(\Delta K^{\prime}>1.0\right)$ companions in the separation range of $0.1-0.2^{\prime \prime}$. However, if we assume that the mass ratio distribution $(q)$ for M8.0-L0.5 stars is similar to that of M0-M4 binaries, then we would expect as least as many binaries with $\Delta K^{\prime}>1.0$ as $\Delta K^{\prime}<1.0 \mathrm{mag}$ Fischer \& Marcy (1992). Although we do not have enough data currently to derive the $q$ distribution for M8.0-L0.5 binaries, we can note that for the 4 systems with separations $>0.2^{\prime \prime}$ we observed an equal number of $\Delta K^{\prime}>1.0$ as $\Delta K^{\prime}<1.0$ mag systems. So it appears reasonable that there should also be an equal number of $\Delta K^{\prime}>1.0$ as $\Delta K^{\prime}<1.0 \mathrm{mag}$ systems in the range $0.1-0.2^{\prime \prime}$. Then based on our detection of 5 systems with $\Delta K^{\prime}<1$ mag with separations of $0.1-0.2^{\prime \prime}$ we would expect to have $\sim 5$ systems with $\Delta K^{\prime}>1.0$ in the range $0.1-0.2^{\prime \prime}$. In reality we detected no systems with $\Delta K^{\prime}>1.0$ with separations $0.1-0.2^{\prime \prime}$. Therefore, to correct for instrumental insensitivity we need to increase the number of binary systems by 5 in the range $0.1-0.2^{\prime \prime}$. Based on this assumption about the mass ratio distribution there should be $\sim 10$ binaries from $0.1-0.2^{\prime \prime}$ when correcting for our instrumental insensitivity. Therefore, the total count for all separations $>0.1^{\prime \prime}$ should be $14 \pm 4$ systems assuming a Poisson error. Therefore, the corrected M8.0-L0.5 binary frequency would be $14 / 39 / 1.92=19 \pm 6 \%$ for separations $>0.10^{\prime \prime}$ or ¿2.6AU.

Hence we have a range of possible binary frequencies from $12 \%$ (in the unlikely case the frequency of systems with $\Delta K^{\prime}>1$ mag with separations $0.1-0.2^{\prime \prime}$ falls to $0 \%$ from the $50 \%$ observed for systems with separations > $\left.0.2^{\prime \prime}\right)$ up to $19 \pm 6 \%$ correcting for insensitivity to tight $0.1-0.2^{\prime \prime}$ systems with faint $\Delta K^{\prime}>1$ companions. In any case, we can state that for systems with separations $a>2.6$ AU the M8.0-L0.5 binary frequency is within the range $19 \pm 7 \%$. Our binary fraction of $19 \pm 7 \%$ is therefore slightly lower than the $32 \pm 9 \%$ observed for (M0-M4) stars with $a>2.6$ AU by Fischer \& Marcy (1992). Hence it appears VLM binaries $\left(M_{t o t}<0.18 M_{\odot}\right)$ ) are slightly less common than (M0-M4) binaries over the whole range $a>2.6 \mathrm{AU}$. This difference may 
disappear once surveys for spectroscopic and even tighter visual VLM binaries are completed.

\subsection{The separation distribution function for M8.0-L0.5 binaries}

The M8.0-L0.5 binaries are very different from M0-M4 stars in the distribution of their semi-major axes (see Figure 1). The M8.0-L0.5 binaries appear to peak at separations $\sim 4 \mathrm{AU}$ (see the histogram in Figure 1) which is significantly tighter than the broad $\sim 30$ AU peak of both the G and M star binary distributions (Fischer \& Marcy (1992), Duquennoy \& Mayor (1991)). Note the peak of Fischer \& Marcy (1992) distribution may shift slightly to smaller values if a few low $(q<0.4 ; \Delta K \lesssim 5 \mathrm{mag})$ binaries were missed in the speckle data used by Fischer \& Marcy (1992) in the range 1-10 AU. But we estimate that this a small effect since very few $(<10 \%) \mathrm{M}$ binaries have $(q<0.4)$ Fischer \& Marcy (1992).

We detect no low mass binary systems wider than $16 \mathrm{AU}$. This cannot be a selection effect since we are highly sensitive to all M8.0-L0.5 binaries with sep $>20-600 \mathrm{AU}$ (even those with $\Delta H>10 \mathrm{mag}$ ). Therefore, we may conclude that M8.0-L0.5 stars likely have just slightly lower binary fractions than $G$ and $M$ stars, but they have significantly smaller semi-major axes on average, and currently no very wide ( $a>20$ AU) systems are known.

More observations of such systems will be required to see if these trends hold over bigger samples. It is interesting to note that in Reid et al. (2001a) an HST survey of $20 \mathrm{~L}$ stars found 4 binaries and a similar binary frequency of $20 \%$. The widest L dwarf binary in Koerner et al. (1999) had a separation of only 9.2 AU. A smaller HST survey of $10 \mathrm{~T}$ dwarfs by Burgasser et al. (2002) found $3 \mathrm{~T}$ binaries and a similar binary frequency of $20 \pm 6 \%$ with no systems wider than 5.2 AU. Therefore, it appears all M8.0-L0.5, L, and T binaries may have similar binary frequencies $\sim 20 \%$ (for $a>3 \mathrm{AU}$ ) and smaller maximum separations (no system wider than $16 \mathrm{AU}$ ) than their more massive $\mathrm{M}$ and $\mathrm{G}$ counterparts.

\subsection{Why are there no wide low mass binaries?}

In Close, Siegler, \& Freed (2002) it is suggested that a strong impulse (or velocity kick) of $\sim 3 \mathrm{~km} / \mathrm{s}$ could explain the lack of any low mass systems with $a>20$ AU. Such a kick could disrupt any VLM/brown dwarf binaries with $a>20$ AU while leaving systems with $a<10$ AU relatively unaffected. This might support recent "ejection" theories of brown dwarf formation from Reipurth \& Clarke (2002) and Bate et al. (2002). However, it is still not clear whether these "ejection" scenarios can reproduce the observed $20 \%$ binary fraction for separations $>2.6$ AU (currently these models produce only $5 \%$ brown dwarf binary fractions Bate et al. (2002)). Future detailed models with more realistic treatments of the individual accretion disks should provide more insight into the plausibility of this formation process. In any case this strong cut-off at $a \sim 20$ $A U$ suggests that VLM/Brown dwarf binaries may form differently (perhaps due to ejection) than higher mass $\left(M_{\text {tot }}>0.185 M_{\odot}\right)$ binaries which are much wider.

Acknowledgments. The Hōkūpa'a AO observations were supported by the University of Hawaii AO group. (D. Potter, O. Guyon, \& P. Baudoz). Support for Hōkūpa'a comes from the National Science Foundation. LMC acknowledges 


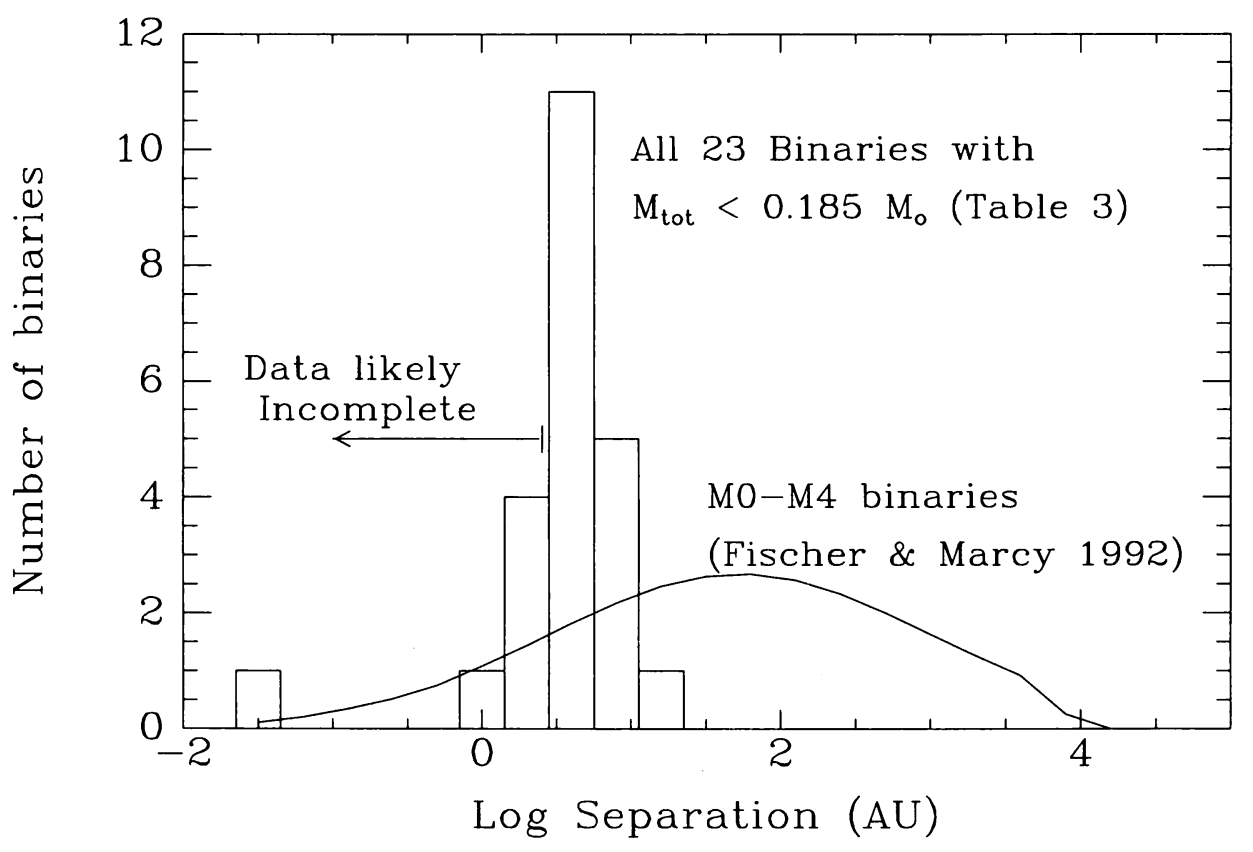

Figure 1. Here we plot (as a histogram) all 23 published VLM binaries $(M 7<S p T<T 6$; Basri \& Martín(1999), Martín, Brandner, \& Basri (1999), Koerner et al. (1999), Reid et al. (2001a), Lane et al. (2001), Potter et al. (2002a), Burgasser et al. (2002), Close, Siegler, \& Freed (2002)) listed in Table 3 of Close, Siegler, \& Freed (2002). These include 12 systems found in our AO study. Note how low mass binaries appear to have smaller separations compared to the M0-M4 binaries of Fischer \& Marcy (1992). Both distributions are normalized to have 23 system in total. We have not tried to correct for instrumental incompleteness. Hence we underestimate the number of VLM binaries with $a<2 \mathrm{AU}$. However, the sharp peak at $4 \mathrm{AU}$ and the lack of any wide $(a>20 A U)$ systems are real features of the distribution and are significantly different from that observed in more massive M0-M4 binaries. Binary systems with $M_{t o t}<0.185 M_{\odot}$ appear quite a bit tighter compared to just slightly more massive M0-M4 binaries. 
support by the AFOSR under F49620-00-1-0294 and from NASA Origins NAG512086. We would also like to send a big mahalo nui to the Gemini operations staff.

\section{References}

Bate, Matthew R.; Bonnell, Ian A.; Bromm, Volker 2002, MNRAS, 332, L65

Reipurth, B. \& Clarke C. 2002, AJ, 122, 432

Burgasser, A.J., Kirkpatrick, J.D, \& Brown, M.E. 2002, BAAS, 34, \#11.05

Burrows, A., Hubbard, W. B., Lunine, J. I., Marley, M. S., Saumon, D. 2000, Protostars and Planets IV (Tucson: University of Arizona Press, eds Mannings, V., Boss, A.P., Russell, S. S.), p. 1339

Basri, G., Martín, E. L. 1999, AJ, 118, 2460

Caloi, V., Cardini, D., D’Antona, F., Badiali, M., Emanuele, A., Mazzitelli, I. 1999, A\&A, 351, 925.

Chabrier, G., Baraffe, I., Allard, F., \& Hauschildt, P. 2000, ApJ, 542, 464

Close, L.M., Potter, D., Brandner, W., Lloyd-Hart, M., Liebert, J., Burrows, A., \& Siegler, N. 2002a, ApJ, 566, 1095.

Close, L.M., Siegler, N., Potter, D., Brandner, W., Liebert, J. 2002b, ApJ, 567, L53.

Close, L.M. Siegler, N., \& Freed, M., 2002, ApJ, submitted.

Dahn C. et al. 2002 ApJ, 124, 1170

Duquennoy, A., Mayor, M. 1991, A\&A, 248, 485

Fischer, D. A., Marcy, G. W. 1992, ApJ, 396, 178

Freed, M., Close, L.M., \& Siegler, N. 2002, ApJL, submitted

Gizis, J.E., Kirkpatrick, J.D., Burgasser, A., Reid, I.N., Monet, D.G., Liebert, J., Wilson, J.C. 2001, ApJ, 551, L163

Gizis, J.E., Monet, D.G., Reid, I.N., Kirkpatrick, J.D., Liebert, J., Williams, R.J. 2000, AJ, 120, 1085

Kirkpatrick, J. Davy; Reid, I. Neill; Liebert, James; Gizis, John E.; Burgasser, Adam J.; Monet, David G.; Dahn, Conard C.; Nelson, Brant; Williams, Rik J. 2000, AJ, 120, 447

Koerner, D. W., Kirkpatrick, J. Davy, McElwain, M. W., Bonaventura, N. R. 1999, ApJ, 526, L25

Lane, B.F., Zapatero Osorio, M.R., Britton, M.C., Martín, E.L., Kulkarni, S.R. 2001, ApJ, 560, 390 
Martín, E.L., Brandner, W., Basri, G. 1999, Science, 283, 5408, 1718

Martín, E. L., Koresko, C. D., Kulkarni, S. R., Lane, B. F., Wizinowich, P. L. 2000 ApJ, 529, L37

Nakajima, T., Oppenheimer, B. R., Kulkarni, S. R., Golimowski, D. A., Matthews, K., Durrance, S. T. 1995, NATURE, 378, 463

Potter, D. et al. 2002a ApJ, 567, 113

Reid, I. Neill; Gizis, John E. 1997a AJ, 113, 2246

Reid, I. Neill; Gizis, John E. 1997b AJ, 114, 1992

Reid, I. N., Gizis, J.E., Kirkpatrick, J.D., Koerner, D. W. 2001a, AJ, 121, 489

Reid, I. N., Burgasser, A. J., Cruz, K. L., Kirkpatrick, J. D., Gizis, J. E. 2001b, AJ, 121,1710

Reid, I. N. et al. 2002, AJ, 124, 519

Siegler, N., Close, L.M., Mamajek, E., Freed, M. 2002, ApJ, in prep

Stetson, P. B. 1987, PASP, 99, 191

van Altena, W.F., Lee, J.T., \& Hoffleit, D 1995, The General Catalog of Trigonometric Stellar Parallaxes (4th ed.; New Haven: Yale University Observatory)

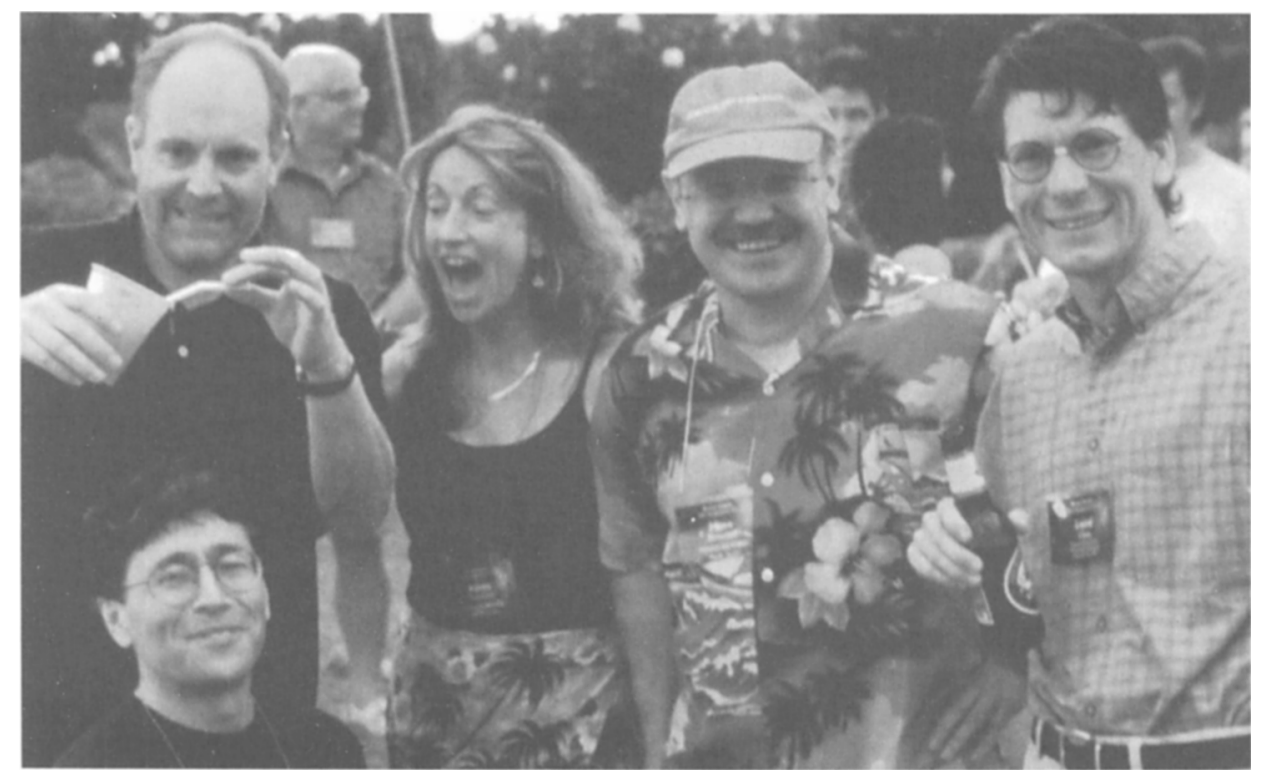

From left to right: John Rayner (kneeling), Mark McCaughrean, Karen Teramura, Hans Zinnecker, and Laird Close. Andrew Pickles is in the background. 